\title{
Diagnosis of Schistosomiasis in Low Endemic Areas
}

\author{
Amal Farahat Allam \\ Alexandria University \\ Egypt
}

\section{Introduction}

Schistosomiasis, also known as bilharziasis is caused by snail transmitted parasites of the genus schistosoma that inhabit the human vasculature.(1,2) It is among the most important parasitic diseases worldwide, with a significant socio economic impact $(3)$. More than 200 million people are infected, and about 200,000 may die from the disease each year. On a global scale, one of thirty individuals has schistosomiasis(4). About 779 million people live in endemic areas in the Middle East, South America, Caribbean, Southeast Asia and particularly sub-Saharan Africa(4). Extreme poverty, the unawareness of the risks, the inadequacy or total lack of public health facilities plus the unsanitary conditions in which millions of people lead their daily lives are all factors contributing to the risk of infection(5).

During the past 20 years, much progress in combating the disease has been achieved based on World Health Organization recommendation strategies. Recent years have seen a notable decrease in the prevalence and morbidity of the disease in many endemic countries $(6,7)$. Many countries of the EMR ( Eastern Mediterranean Region) namely Egypt, Iraq, Syria, Libya, Oman and Saudi Arabia have now reached low schistosomiasis endemicity. For instance, the overall prevalence of schistosomiasis in Egypt, was about 40\% in (1967) before the national control program started by WHO (EMRO). In 2006, due to different control measures, the overall prevalence fell down to $<3 \%$. However there are still hot spot transmission foci with prevalence rate about $10 \%$. Therefore lack of well structured prevention, control and elimination programs could lead to emergence or resurgence of the controlled disease. Schistosomiasis has been eliminated in Iran, Lebanon, Morocco and Tunisia, no new cases were detected over the past few years. Furthermore, WHO adopted a resolution calling on countries in low transmission areas, to sustain successful control activities in order to eliminate schistosomiasis(8) . In these conditions, where elimination of schistosomiasis is aimed for, case detection may pose a problem because the commonly used methods for the assessment of infection may lack necessary sensitivity to accurately determine the prevalence of schistosomiasis or of parasite burden $(9,10)$. $\mathrm{WHO}(2001,2006)(11,12)$ asked for sensitive assays for active surveillance, specially in situations with no or very low transmission.

Notwithstanding the large number of tests for diagnosing schistosomiasis, few have withstood tests of effectiveness, reproducibility, cross reactivity and predictive values (Rabello et al. 2002)(13). The diagnostic strategies traditionally rely on the detection of parasite eggs in stool (S. mansoni, S.japonicum) or urine (S. haematobium), antibody and 
antigen detection techniques. Lier et al (2008)(14) reported that there is no satisfactory "gold standard" diagnostic test in low intensity infections.

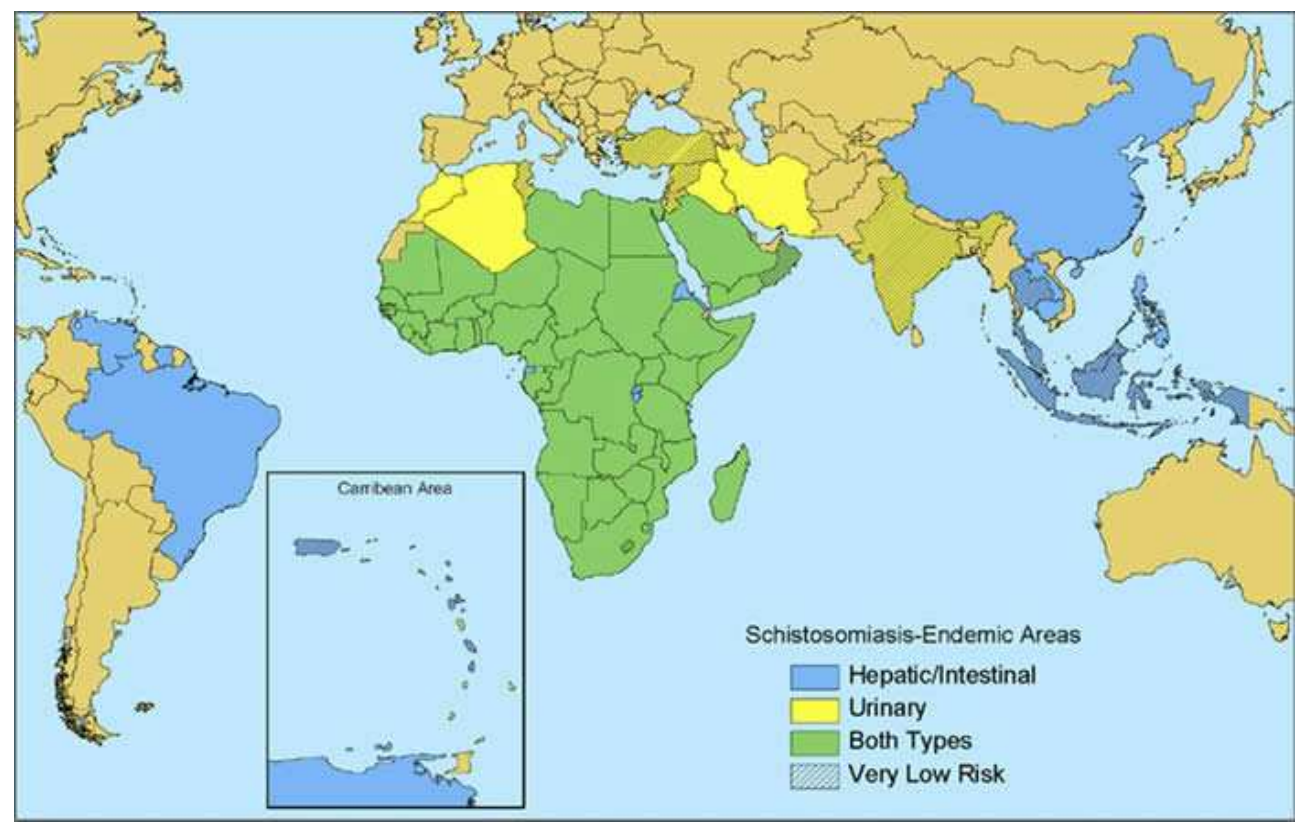

Fig. 1. Map showing Schistosomiasis Endemic Areas

The Kato-Katz technique (15) is currently the most used method for fecal examination because it is quantitative, relatively inexpensive simple and fast. However, due to the small amount of stool examined (less than $50 \mathrm{mg}$ ), its sensitivity is logistically prohibitive to monitor regularly or comprehensively low or moderate infections. Due to random distribution effects, the analyzed sample may not contain eggs even if the disease is active. It is thus very difficult to achieve a conclusive confirmation of successful therapy. A significant increase in the sensitivity of the method is gained by microscopic examination of multiple samples, but this is a limiting procedure for field work(16,17).

To overcome the current limitations with respect to diagnosis, the simultaneous use of different diagnostic methods, such as antibody detection followed by stool examination of seropositive individuals, has been applied to monitor the human population and to identify the small number of infected people once morbidity control is achieved. Dias et al. (1989)(18) reported that in low endemic areas for schistosomiasis, serologic prevalence indices have shown to be 2 to 5 times higher than the parasitologic indices. In Pedro de Toledo, the prevalence was $55.5 \%$ by the IgG, and it was $22.8 \%$, by Kato-Katz method, with an infection intensity of $58.5 \mathrm{epg}$. Thus, the serologic prevalence was 2.6 times higher than the parasitologic prevalence. Noya et al(1999)(19) reported that the serologic prevalence by IgG was $33.2 \%$, which was 20 times higher than the parasitologic prevalence of $1.6 \%$, obtained by Kato-Katz method, with an infection intensity of 40.9 epg.

Burlandy- Soares et al (2003)(20) reported that the high difference between serologic and parasitologic prevalence data is undoubtedly due to the low diagnostic sensitivity of the 
parasitologic methods. Other might be the presence of individuals who have developed immune response to $S$. mansoni antigens after exposure to cercariae, but without development of infection, or have been cured after chemotherapy and still present circulating antibodies. It is a well known fact that immediately after chemotherapy, the levels of specific antibodies rise stimulated by the death of the parasites and fall after. Although the serologic techniques (ELISA, IHA and IFT) are very efficient, they do not constitute part of the diagnostic routine for schistosomiasis, and also they are not commonly used in epidemiologic studies. Moreover, antibody detection methods often cannot distinguish between current and past infection and may also present a high level of cross-reactivity. Blood collection is not easily applicable under field conditions, therefore this method is not recommended for field studies in endemic areas (Gryseels et al.,2006)(21). However, Kanamura et al (1998)(22) and Burlandy-Soares et al (2003)(20) reported that the introduction of serologic methods in epidemiological studies, identifying potential infection sources, may contribute towards the reduction of residual transmission. This procedure could be included in a national program for the control of schistosomiasis mansoni.

Antigen based assays, such as circulating cathodic antigen (CCA) detection in urine and circulating anodic antigen (CAA) in serum, have proven to be a valuable, field applicable method. Unfortunately, in low intensity of infection, most of the positive antigen levels were very close to the cut off value, which is a disadvantage for the epidemiological application of the assay. It was concluded that for the diagnosis of schistosomiasis in low endemic areas, circulating antigen detection should be considered as a complementary tool rather than a substitute for parasitological examination specially that the detection of CCA in urine and CAA in serum supplies additional information to determine more accurately the "true" prevalence of infection(23,24).

To date, Percoll sedimentation technique was established as the technique of choice for diagnosis of $S$. mansoni in low intensity conditions and post treatment ${ }^{(25)}$. This method always gives better and more consistent estimate than the Kato Katz , and efficiently detects low intensity infections up to 4 eggs /gm. Amongst Kato-Katz negative Schistosoma mansoni specimens in a hypoendemic area with a prevalence of 5\%, Percoll detected $11 \%$ positive cases and $7 \%$ post treatment. The efficiency of Percoll is based on the fact that it depends on $250 \mathrm{mg}$ feces; one egg in the sediment would mean 4eggs/gm, whilst three Kato slides can diagnose up to 8 epg when only one egg is detected in one of the three slides examined. Moreover, detection of one to two eggs per fecal smear could be missed more easily within the fecal debris of Kato-Katz than the clear fields of the Percoll technique. Similar findings were reported by Eberl et al. (2002)(26), who found that the triple Kato-Katz examination on consecutive samples still missed $7.4 \%$ of all patients, whereas low egg numbers were detected efficiently only by Percoll technique.

Pontes et al. (2002)(27) reported the first use of PCR for the diagnosis of S. mansoni DNA in fecal samples. PCR methods have shown high sensitivity and specificity for the detection of S. mansoni DNA in stool samples, yet PCR gave negative results among few cases considered sure positives by microscopy $($ tables1,2). Similar results were obtained by many authors $(13,25,28,29,30)$.

Table (1) shows that the analysis of discordant results revealed that 15 samples were positive only by the PCR and two samples were positive only by Kato Katz technique. 


\begin{tabular}{|lccc|}
\hline \multicolumn{4}{c|}{ Kato-Katz faecal examination } \\
\hline Positive & Negative & Total \\
\hline PCR & & & \\
Positive & 26 & 15 & 41 \\
Negative & 2 & 24 & 26 \\
Total & 28 & 39 & 67 \\
\hline
\end{tabular}

Kappa index: 0.511 (Gomes et al 2009)(30).

Table 1. Comparative evaluation of the PCR and the Kato-Katz technique for the diagnosis of Schistosoma mansoni infection

\begin{tabular}{|lccc|}
\hline \multicolumn{3}{c|}{ Percoll } \\
\hline PCR & Positive & Negative & Total \\
Positive & & & \\
Negative & 2 & 16 & 18 \\
Total & 7 & 52 & 59 \\
\hline
\end{tabular}

Kappa index $=0.009, \mathrm{P}>0.05$ no agreement (Allam et al 2009)(25)

Table 2. Agreement of the Percoll and PCR for the detection of Schistosoma mansoni cases negative after Kato-Katz technique

Comparing the results of the 77 cases analyzed by PCR with their results by Percoll revealed that only two patients gave concordant positive results; seven cases positive by Percoll were negative by PCR (Table2).

Pontes et al. (2003)(25) reported that missed cases after PCR were certainly misdiagnosed by the DNA amplification assay due to many factors such as: inhibition of the amplification reaction by fecal compounds and / or DNA degradation during transportation from the field, variation in egg output and uneven distribution in feces.

Oliveira et al.(2010)(31) reported that PCR can be an important tool for detecting S. mansoni infection in individuals excreting few eggs in feces. Moreover, the determination of the infection through the detection of $S$. mansoni DNA in stool samples from seropositive individuals represents a new means of confirming the results of IgG-ELISA for schistosomiasis. Therefore, studies in this direction should be encouraged and extended. Also, PCR have shown promise in the detection of parasites in a variety of media. Qualitative techniques have been developed to detect the presence or absence of $S$. jabonicum and avian schistosome cercariae recovered from snail hosts, as well as S. mansoni cercariae in water(32).

It was reported that conventional PCR requires several steps after DNA amplification, including electrophoresis or blotting and hybridization, which are limited in the number of samples that can be conveniently analyzed. A multiplex real-time PCR assay for the detection and quantification of S. mansoni and Schistosomal DNA in faecal samples was developed and evaluated as an alternative diagnostic method to study the epidemiology of schistosomiasis(33). Primers and probes targeting the cytochrome $\mathrm{c}$ oxidase gene were designed for species-specific amplification and were combined with an internal control. Using positive control DNA extracted from adult Schistosoma worms and negative control samples $(n=150)$ with DNA from a wide range of intestinal microorganisms, the method 
proved to be sensitive and $100 \%$ specific. For further evaluation, duplicate stool specimens with varying $S$. mansoni egg loads were collected in northern Senegal from pre-selected individuals $(n=88)$. The PCR cycle threshold values, reflecting parasite-specific DNA loads in faeces, showed significant correlation with microscopic egg counts both for S. mansoni in stool and S. haematobium in urine. Although eggs of S. haematobium were not observed in stool samples, the presence of $S$. haematobium DNA was detected successfully. The Schistosoma detection rate of PCR $(84.1 \%)$ was similar to that of microscopy performed on duplicate stool samples (79.5\%). The simple fecal sample collection procedure and the high throughput potential of the multiplex real-time PCR provide a powerful diagnostic tool for epidemiological studies on schistosomiasis in remote areas. Moreover, it demonstrates high sensitivity, even in samples containing a single schistosoma egg in stool(33,43).

When comparing the real-time PCR with microscopy-based assays, the PCR is less labour intensive when many samples are to be examined. The PCR method also has the advantage of being less influenced by observer variation/bias as compared to microscopy-based methods.

More recently, Gomes et al (2006)(30) ,Hung et al (2008)(32)and lier et al (2009)(34) established and developed SYBR Green ( a fluorescent based technology) and Taq Man probe real time PCR assays for the detection and quantification of schistosomal DNA. They reported that they are potentially useful for quantification of parasite burden in human infection.

Gomes et al (2010)(35) reported development of the PCR-ELISA system for the detection of Schistosoma DNA in human feces as an alternative approach to diagnose light infections. The system permits the enzymatic amplification of a specific region of the DNA from minute amounts of parasite material. Using the proposed PCR-ELISA approach for the diagnosis of a population in an endemic area in Brazil, 30\% were found to be infected, as compared with the $18 \%$ found by microscopic fecal examination. The genus specificity of PCR-ELISA was assessed with purified DNA from S. mansoni, S. haematobium, S. bovis, S. intercalatum, S. japonicum, S. magrebowiei and S. rhodaini adult worms. Considering the results obtained with the Schistosoma PCR-ELISA system based on two levels of intensity of infection (1-100 epg and >100 epg), according to the Kato-Katz stool examination method, the assay revealed the potential to be considered semi-quantitative, as the mean absorbance readings corresponded to the intensity of infection. The high sensitivity of the Schistosoma PCR-ELISA system was evidenced by the high number of positive samples in both groups. The advantage of PCRELISA as compared to PCR-electrophoresis is that it makes use of standard equipment widely used for the processing of ELISAs, and the reagents used are easy to obtain commercially. Therefore, PCR-ELISA allows for the use of PCR-based DNA diagnosis for routine purposes in laboratories in less developed countries with fewer resources.

Nowadays, we have many sensitive methods for schistosomiasis detection in areas of low endemicity. Percoll sedimentation technique as parasitologic test of choice. Conventional PCR as a molecular technique for detection of schistosoma eggs. It can be used to confirm the results of seropositives through the detection of schistosomiasis in stool samples. Real time PCR assays for detection and quantification of schistosomiasis. PCR ELISA as a semiquantitative technique for diagnosis of low intensity of schistosomiasis.

Thus, as the prevalence and intensity decrease, the benefit of increased sensitivity using the molecular tests must be weighed against additional costs. Although the techniques require a complex laboratory infrastructure and specific funding it may be used by control programs targeting the elimination of schistosomiasis( Lier et al 2009)(34). 


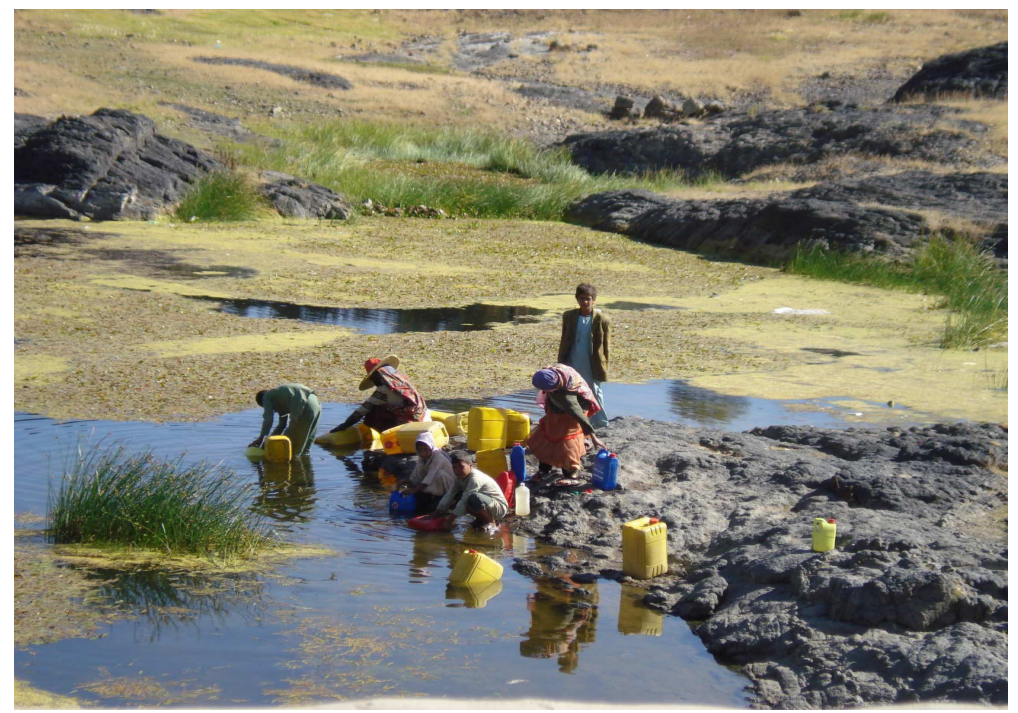

Fig. 2. Can schistosomiasis be eliminated? Fenwick et al(5).

\section{References}

[1] WHO (2011). Epidemiological records. 86: 73-80.

[2] Lier T, Simonsen GS, Wang T, Lu D, Haukland HH, Vennervald BJ, Hegstad J, and Johansen MV (2009). Real Time Polymerase Chain Reaction for Detection of low intensity Schistosoms japonicum infections in China. Am J Trop Med Hyg 81: 428- 432.

[3] World Health Organization (2008). The social context of schistosomiasis and its control: an introduction and annotated bibliography.

[4] Chitsulo L, Loverde P, Engels D (2004). Schistosomiasis. Nat Rev Microbiol 2: 12-13.

[5] Fenwick A et al (2003). Drugs for the control of parasitic diseases: current status and development in schistosomiasis. Trends parasitol 19:509-15.

[6] Lammie PJ, Fenwick A, Utzinger J (2006) A blueprint for success: integration of neglected tropical disease control programmes. Trends Parasitol 22: 313-321.

[7] King CH (2009). Toward the elimination of schistosomiasis. N Engl J Med 360: 106-109.

[8] WHO (2007). Report of inter country meeting on strategy to eliminate schistosomiasis from the Eastern Mediterranean Region. Muscat, Oman . 6-8 November.

[9] Utzinger J, Zhou XN, Chen MG, Bergquist R (2005) .Conquering schistosomiasis in China. Acta Trop 96: 69-96.

[10] World Health Organization (2005) .Report of the scientific working group meeting on Schistosomiasis. Geneva, 14-16 November.

[11] WHO (2001). Report of the World Health Organization informal consultation on schistosomiasis in low transmission areas: control strategies and criteria for elimination. 10-13. WHO/CDS/CPE/SIP/2001.

[12] WHO (2006). World Health Organization Special Programme for Research and Training in Tropical Diseases. TDR home page . at.www. who.int/tdr. 
[13] Rabello A, Pontes LA \& Dias-Neto E (2002) Recent advances in the diagnosis of Schistosoma infection: the detection of parasite DNA. Mem Inst Oswaldo Cruz 97:171-172.

[14] Lier T, Johansen MV, Hjelmevoll SO, Vennervald, Simonsen GS (2008). Real time PCR for detection of low intensity Schistosoma japonicum infections in a pig model. Acta Trop 105-: 74-80.

[15] Katz N, Chaves A \& Pellegrino J (1972) A simple device for quantitative stool thick smear technique in Schistosoma mansoni Revista do Instituto de Medicina Tropical de Sao Paulo 14:397-400.

[16] Yu JM, de Vlas SJ, Yuan HC, Gryseels B (1998) Variations in fecal Schistosoma japonicum egg counts. Am J Trop Med Hyg 59: 370-375.

[17] Utzinger J, N'Goran EK, N'Dri A, Lengeler C, Xiao S, et al. (2000) Oral artemether for prevention of Schistosoma mansoni infection: randomized controlled trial. Lancet 355: 1320-1325.

[18] Dias LCS, Kawazoe U, Glasser CM, Hoshino-Shimizu S, Kanamura HY, Cordeiro JA, Guarata OF, Ishirata GJ (1989). Schistosomiasis mansoni in the municipality of Pedro de Toledo (São Paulo, Brazil) where the Biomphalaria tenagophila is the snail host: I. Prevalence in human population. Rev Inst Med Trop 31: 110 -118.

[19] Noya BA, Balzan C, Artega C, Cesari I, Noya O (1999). The last fifteen years of schistosomiasis in Venezuela: features an evolution. Mem Inst Oswaldo Cruz 94: 136-146.

[20] Lanny Cristina Burlandy-Soares Luiz Cândido de Souza Dias, Hermínia Yohko Kanamura, Edward José de Oliveira, Ricardo Mario Ciaravolo (2003). Schistosomiasis mansoni: Follow-up of Control Program Based on Parasitologic and Serologic Methods in a Brazilian Community of Low Endemicity. Mem Inst Oswaldo Cruz 98: 853-859

[21] Gryseels B, Polman K, Clerinx J, Kestens L (2006) Human schistosomiasis. Lancet 368: 1106-1118.

[22] Kanamura HY, Dias LCS, Glasser CM, Silva RM, Patucci RMJ, Chiodelli SG, Addis DG (1998). Detection of IgM antibodies to Schistosoma mansoni gut-associated antigens for the study of the dynamics of schistosomiasis transmission in an endemic area with low worm burden. Rev Inst Med Trop São Paulo 40: 225-231.

[23] El-Morshedy H, Kinosien B, Barakat R, Omer E, Khamis N, Deelder AM, Phillips M (1996). Circulating anodic antigen for detection of Schistosoma mansoni infection in Egyptian patients. Am J Trop Med Hyg 54: 149-153

[24] Van Dam GJ, Wichers JH, Ferrera TM, Ghati D, Van Amerongen A, Deelder AM (2004 ). Diagnosis of schistosomiasis by reagent strip test for detection of circulating cathodic antigen. J Clin Microbiol. 42: 5458-5461.

[25] Allam AF, Kader O, Zaki A, Shehab AY, Farag HF (2009). Assessing the marginal error in diagnosis and cure of Schistosoma mansoni in areas of low endemicity using Percoll and PCR techniques. Trop Med Int Health 14: 316-321.

[26] Eberl M, Al-Sherbiny M, Hagan P, Ljubojevic S, Thomas AW \& Wilson RA (2002). A novel and sensitive method to monitor helminth infections by faecal sampling. Acta Tropica 83: 183- 187. 
[27] Pontes LA, Oliveira MC, Katz N, Dias-Neto E\&Rabello A (2003). Comparison of polymerase chain reaction and the Kato-Katz technique for diagnosing infection with Schistosoma mansoni. Am J Trop Med Hyg 68:652- 656.

[28] Pontes LA, Dias-Neto E \& Rabello A (2002). Detection by polymerase chain reaction of Schistosoma mansoni DNA in human serum and feces. Am J Trop Med Hyg 66: 157-162.

[29] Hamburguer J, Turetski T, Kapeller I \& Deresiewi CZR (1991). Highly repeated short DNA sequences in the genome of Schistosoma mansoni recognized by a species specific probe. Mol Biochem Parasitol 44:73-80.

[30] Gomes L I, Marques L HS , Enk M J, Coelho P M Z, Rabello A(2009). Further evaluation of an updated PCR assay for the detection of Schistosoma mansoni DNA in human stool samples Mem Inst Oswaldo Cruz 104: 1194-1196

[31] Oliveira LM, Santos HL, Gonçalves MM, Barreto MG, Peralta JM (2010). Evaluation of polymerase chain reaction as an additional tool for the diagnosis of low-intensity Schistosoma mansoni infection. Diagn Microbiol Infect Dis 68:416-21.

[32] Hung YW, Remais J (2008). Quantification and detection of Schistosoma japonicum cercariae in water by real time PCR. Plos Neg Trop Dis 11: 337- 348.

[33] Ten Hove RJ, Verweij JJ, Vereecken K, Polman K, Dieye L, van Lieshout L (2008). Multiplex real-time PCR for the detection and quantification of Schistosoma mansoni and S. haematobium infection in stool samples collected in northern Senegal. Trans R Soc Trop Med Hyg 102:179-85.

[34] Lier T, Simonsen GS, Wang T, Lu D, Haukland HH, Vennervald BJ, Hegstad J, and Johansen MV (2009). Real Time Polymerase Chain Reaction for Detection of low intensity Schistosoms japonicum infections in China. Am J Trop Med Hyg 81,428432.

[35] Gomes LI, Marques LHdS, Enk MJ, de Oliveira MC, Coelho PMZ, et al. (2010) Development and Evaluation of a Sensitive PCR-ELISA System for Detection of Schistosoma Infection in Feces. PLoS Negl Trop Dis 4(4): e664. 


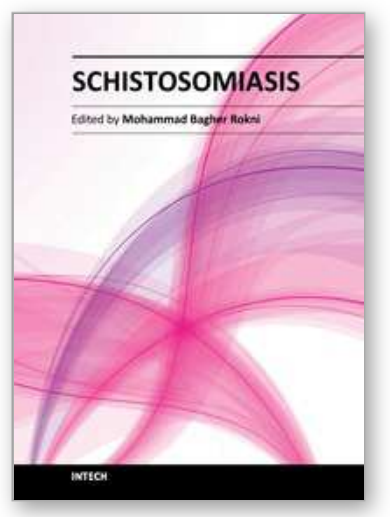

\author{
Schistosomiasis \\ Edited by Prof. Mohammad Bagher Rokni
}

ISBN 978-953-307-852-6

Hard cover, 310 pages

Publisher InTech

Published online 13, January, 2012

Published in print edition January, 2012

In the wake of the invitation by InTech, this book was written by a number of prominent researchers in the field. It is set to present a compendium of all necessary and up-to-date data to all who are interested. Schistosomiasis or blood fluke disease, also known as Bilharziasis, is a parasitic disease caused by helminths from a genus of trematodes entitled Schistosoma. It is a snail-borne trematode infection. The disease is among the Neglected Tropical Diseases, catalogued by the Global Plan to combat Neglected Tropical Diseases, 2008-2015 and is considered by the World Health Organization (WHO) to be the second most socioeconomically devastating parasitic disease, next to malaria. WHO demonstrates that schistosomiasis affects at least 200 million people worldwide, more than 700 million people live in endemic areas, and more than 200.000 deaths are reported annually. It leads to the loss of about 4.5 million disability-adjusted life years (DALYs).

\title{
How to reference
}

In order to correctly reference this scholarly work, feel free to copy and paste the following:

Amal Farahat Allam (2012). Diagnosis of Schistosomiasis in Low Endemic Areas, Schistosomiasis, Prof. Mohammad Bagher Rokni (Ed.), ISBN: 978-953-307-852-6, InTech, Available from:

http://www.intechopen.com/books/schistosomiasis/diagnosis-of-schistosomiasis-in-low-endemic-areas

\section{INTECH}

open science | open minds

\author{
InTech Europe \\ University Campus STeP Ri \\ Slavka Krautzeka 83/A \\ 51000 Rijeka, Croatia \\ Phone: +385 (51) 770447 \\ Fax: +385 (51) 686166 \\ www.intechopen.com
}

\author{
InTech China \\ Unit 405, Office Block, Hotel Equatorial Shanghai \\ No.65, Yan An Road (West), Shanghai, 200040, China \\ 中国上海市延安西路65号上海国际贵都大饭店办公楼405单元 \\ Phone: +86-21-62489820 \\ Fax: $+86-21-62489821$
}


(C) 2012 The Author(s). Licensee IntechOpen. This is an open access article distributed under the terms of the Creative Commons Attribution 3.0 License, which permits unrestricted use, distribution, and reproduction in any medium, provided the original work is properly cited. 